\title{
A QUALITY-OF-SERVICE (QOS) BASED APPROACH FOR THE COMMUNICATION SUPPORT IN NETWORK-BASED CONTROL SYSTEMS: AN ON-GOING PROJECT
}

\author{
Ricardo Alexandre Reinaldo de Moraes ${ }^{1}$ and Francisco Vasques $^{2}$ \\ ${ }^{1}$ Department of Exact and Technological Sciences, UNIPLAC, Planalto Catarinense University \\ Av. Castelo Branco, 170 Lages SC, Brasil \\ 2 Department of Mechanical Engineering, FEUP, University of Porto \\ Rua Dr. Roberto Frias, 4200-465 Porto, Portugal \\ e-mail: ricardo@uniplac.net,vasques@fe.up.pt
}

\begin{abstract}
:
Due to the increased availability of low cost network technology, the implementation of Network-based Control Systems (NCS) is becoming widely accepted. Current NCS are usually supported by small-dedicated communication networks (known as fieldbus networks) operating in access-controlled mediums, which are able to guarantee the delivery of control data within known and bounded delays.

It is however foreseeable that, access-uncontrolled networks (such as packet-based networks) will play an important role in future NCS. In this paper, we present an ongoing project, proposing the use of a QoS architecture to implement NCS built upon shared networks with uncontrolled access, i.e., considering communication environments subject to unknown disturbances.

Within this project, several QoS architectures for packet-based networks are looked at carefully, such as the "IntServ" and the "DiffServ" architectures and the "Multi-Protocol Label Switching" (MPLS) and the "Constraint Based Routing" strategies. Such multiple QoS architectures will be evaluated through the use of an experimental platform enabling the implementation and evaluation of network-based control systems
\end{abstract}

Keywords: Network-based Control Systems, Quality of Service, Manufacturing Systems.

\section{INTRODUCTION}

Recently, important technological advances can be observed in the development of devices with sensorial and communication capabilities, able to be ubiquitously incorporated into the physical world. Examples of such devices are the microelectromechanical based sensors (MEMS-based sensors) used in automobile systems, smart homes, large manufacturing systems, intelligent highways and networked city services. Generally, such devices are characterized by the combination of reduced cost $v s$. large communication and processing capacity.

The advent of ubiquitous, distributed computation, communication, and sensing systems has begun to create an environment in which we have access to enormous amounts of data and the ability to process and communicate that data in ways that were unimagined 20 years ago (Murray et al., 2003). This will have a huge impact in applications where computer systems strongly interact with the environment, enabling the true implementation of the pervasive distributed computing paradigm. Example of application domains where such paradigm will have an important impact vary from automobile systems to smart homes, smart cities and highways, large production systems and their logistics chains. Within such context, the technological landscape will be highly influenced by state-of-the-art research in the domains of convergence between communications, computing, and control.

Control systems are fundamental elements for the construction of complex computer systems characterized by a close interaction with the exterior environment. Actually, one of the main targets when designing complex computer systems is the optimization of both its performance and reliability in the presence of unexpected disturbances from the outside environment. Such target can be considered similar to that one of a traditional control system, i.e. its capability to provide an adequate service in the presence of unexpected disturbances.

The implementation of network-based feedback control systems is becoming widely accepted, due to both economical reasons (inexpensive devices) and the ability to decentralize control functions. Such type of feedback control implementation, where the control loop is closed through a real-time network, is referred as Network-based Control System (NCS).

In a NCS, a timely and robust communication service is fundamental for the adequate behavior of the supported control application. It is well known that, 
either lost or dropped control messages or unmodeled (and variable) communication delays are difficult to tolerate in current control systems.

Current NCS are usually supported by smalldedicated communication networks (known as fieldbus networks) operating in access-controlled mediums, which are able to guarantee the delivery of control data within known and bounded delays.

It is however foreseeable that, access-uncontrolled networks (such as packet-based networks) will play an important role in future NCS. When dealing with such timely unpredictable networks, two options can be followed:

a) either a new theory/practice for network-based control systems is developed for systems operating in distributed, asynchronous and packet-based environments;

b) or new methodologies for supporting real-time control communications in such kind of networks are devised.

In this paper, we present the main guidelines of an on-going project, which proposes the use of a QoS architecture to implement NCS built upon shared networks with uncontrolled access, i.e., considering the communication environment subject to unknown disturbances.

Within this project, several QoS architectures for packet-based networks are looked at carefully, such as the "IntServ" and the "DiffServ" architectures and the "Multi-Protocol Label Switching" (MPLS).

Such multiple QoS architectures will be evaluated through the use of an experimental platform, which enables the implementation and evaluation of real NCS. Such platform enables the performance evaluation of both the case of NCS supported by local networks (LANs) or supported by public data communication networks. A special emphasis is given to manufacturing systems that currently use synchronous communication services to support NCS.

This paper is organized as follows. In Section 2 the major challenges concerning the implementation of the pervasive distributed computing paradigm are explained.

In Section 3, several QoS architectures for packetbased networks are looked at carefully, such as the "IntServ" and the "DiffServ" architectures and the "Multi-Protocol Label Switching" (MPLS).

Finally, in Section 4, an experimental platform intended for the experimental evaluation of multiple QoS architectures is described. Such experimental platform enables the performance evaluation of either the case of NCS supported by local networks (LANs) or the case of NCS involving the use of public data communication networks.

\section{NETWORK-BASED COMPUTING SYSTEMS}

Carrying out complex computer systems implementing the pervasive distributed computing paradigm requires a great research effort on new application domains, mainly in research domains in the intersection between Computer Science, Control
Theory, and Communication Systems. The major challenges concerning such implementations have been pointed out (Murray et al., 2003) as being:

a) Control in distributed, asynchronous, networked environments, namely for the cases of complex computer systems characterized by a close interaction with the outside environment and interconnected through packet-based communications. This is especially true in applications where one cannot ignore computational and communication constraints in performing control operations.

b) Building very reliable systems from unreliable components, able to prevent the global system damage, even in the case of individual component failures. This aspect is especially relevant for the case of control applications, where the system must automatically reconfigure itself in case of individual component failures, so that its performance degrades gradually and not abruptly

Thus, these new systems to be developed must take into consideration the QoS (quality of service) obtained. This model must count on features such as shared resources, high availability, end-to-end requests, and adaptation (follow the environment status and get adapted automatically) (Schmidt et al., 2000; Pedatella et al., 2003). There are many applications of crucial security, where the interruption of a service due to failure can produce important damage or losses. Therefore, fault tolerance becomes a requirement of fundamental importance on these systems, once a fault tolerant system keeps running even with failure to its components.

\subsection{Control in Communication Systems vs. Communication in Control Systems}

The crossroad between Computer Science, Control Theory, and Communication Systems is precisely one of the research domains that have been identified as one of the most promising I\&D areas for future developments in the scope of control systems (Murray et al., 2003)

The implementation of pervasive distributed systems by means of components ubiquitously incorporated into the physical world, presents an unquestionable attractive for large-scale complex systems control. The two main open research domains in the scope of this area of convergence are: the control of largescale communication systems and the network-based control systems.

The control of large-scale communication systems includes topics related to congestion control, routing, information management and power management. The fundamental aspects to consider are related to the large dimension of the systems, its decentralised nature, the difficulty inherent to its stabilization (due to the delays the control actions are subject to) and the uncertainties associated with its peculiar parameters: traffic demand, network topology, transmission channel characteristics, etc. 
On the other hand, network-based control systems have been assuming a more relevant role in the context of control systems, due to the alwaysincreasing number of systems made up of a large number of components ubiquitously incorporated in the physical world. Due to the continuous evolution of communication systems, as well in economical terms as in working reliability, it can be predicted that, in the near future, even small-located control systems will become network-based. In other words, it will soon be trivial to come across data related to sensors, actuators, diagnose functions, command functions and co-ordination functions being transferred in real time through communication networks.

One of the major advantages of such network-based implementations, where the control functions are distributed throughout multiple computer systems, is the ability to improve the reliability of the global system operation, through the implementation of redundancy.

\subsection{Target System}

Presently, NCS are still supported by synchronous communication services, which require the use of specific communication protocols in controlled communication environments able to guarantee the information transfer in upper bounded time intervals. The problem will be much more severe if noncontrolled communication environments, i.e., when uncontrolled interference between control traffic and other traffics is considered. Thus, innovative methodologies must be considered to deal with this problem.

It is also important to realize that, whatever the communication environment under consideration, its robust operation is bounded to either the weakest individual component of the distributed computing system, or to the possibility of cascade damages that can provoke the system breakdown, in terms of performance and/or running reliability.

In this paper, we propose the use of a QoS architecture to implement a NCS built upon shared packet-based networks with uncontrolled access, i.e., considering communication environments subject to unknown disturbances. Therefore, several QoS architectures for packet-based networks will be looked at carefully, such as the "IntServ" and the "DiffServ" architectures and the "Multi-Protocol Label Switching" (MPLS)" strategies.

Such multiple QoS architectures will be evaluated through the use of an experimental platform enabling the implementation and evaluation of network-based control systems (NCS). This experimental platform enables the performance evaluation of both the cases of NCS supported by local networks (LANs) or involving the use of public packet-based data communication networks. A special emphasis will be given to manufacturing systems that currently use synchronous communication services to support NCS.

\section{OPEN PROBLEMS}

The implementation of a network-based control system, within which the logical ring among sensors, controllers and actuators is closed through a communication network, requires a real-time communication service guaranteeing that (Martí et al., 2001):

a) the communication delay has an upper bounded and predictable value, when a previously defined set of load assumptions is considered;

b) the jitter associated either to the sampling or the actuation delay, an upper bounded and predictable value, when a previously defined set of load assumptions is considered;

c) the rate of message delivery failure is smaller than an upper bounded value, when a previously defined set of failure assumptions is considered.

Clearly, the considered upper bound for each of these three items must be in accordance with the characteristics of the supported control systems, namely with their control cycle time and their robustness to control data losses. Namely, the maximum jitter associated to the sampling or the actuation delay must be carefully quantified, as the system performance can be strongly influenced by an exceeding value for one of these parameters.

\subsection{Real-Time Communication Concepts}

Conceptually, a real-time communication service can be provided by any communication system, given its ability to support the implementation of real-time channels. Such real-time channels concept (Ferrari et al., 1990) has been proposed as a mean to provide upper bounded delays to specific connections in conventional packet-switched networks. This concept was later refined and extended in (Kandlur et al., 1994). According to the definition, each packet is assigned a deadline over each link on its route and the packet transmission over a link is scheduled according to its deadline. Using an adequate deadline assignment policy, the network will deliver the packets guaranteeing its timing requirements.

The fundamental problem for a real-time communication service resides on the capability that a specific communication (or routing) protocol will have to support the implementation of real-time channels. The following problems will have to be solved for any communication (or routing) protocol under consideration (Zheng and Shin, 1998):

a) The schedulability problem: Given a set of realtime channels supported by a communication link, there is the need to verify if all packets travelling through these channels can be delivered before their deadlines.

b) The minimum guaranteed delay problem: Given a new real-time channel to be established, calculate the minimum delivery delay upper bound that a link can guarantee, without violating the timing guarantees of the already established real-time channels. 
The implementation of real-time channels on communication systems, aiming to offer real-time communication services, is a complex problem.

In the following subsections, a non-exhaustive presentation of some of the more relevant protocols that must be explored in the current context is given.

\section{Packet-Based Communication Protocols to BE EXPLORED}

The demand for services with different quality levels within the Internet brought the development of new service models, within which the application requirements can be specified. However, the service provided today on the Internet is based in the best effort paradigm, i.e., it does not guarantee a real-time service to applications, bearing only a compromise of trying to timely deliver the information sent by the emitter to the receiver.

The quality of transport offered to the packet flows is usually known as Quality of Service (QoS). The QoS concept in communication networks is a fundamental concept, as it clearly characterizes the provided services and the associated guarantees. For instance, each provided service must be described by a set of parameters and its execution must be in conformity with the service specification. For example, a service with an upper bound of $15 \mathrm{~ms}$ for the delay time can deliver information with a $5 \mathrm{~ms}$ delay, but never with a $16 \mathrm{~ms}$ delay.

An adequate management of the elements involved in the QoS negotiation is required, in order to guarantee a consistent behavior of the communication system. For instance, the addition of a new router must not compel a service with a $15 \mathrm{~ms}$ delay to be provided with a $16 \mathrm{~ms}$ delay. Thus, the adequate management of communication networks with QoS must consider not only the best effort paradigm, but also QoS requirements (Granville, 2001).

The use of the TCP/IP architecture to support data communication with timing requirements (deadlines) triggered several research works about QoS issues. The creation by the IETF of several work groups directed to the use of QoS on TCP/IP networks (IETF, 2002) had a consolidation effect on the ongoing research works about QoS issues.

Currently, several technologies offer QoS in communication services, pointing out among them: the Integrated Service model (IntServ) and the Differentiated Service model (DiffServ); Traffic Engineering, Multi-Protocol Label Switching (MPLS); and Constraint Based Routing (Wang, 2001).

On the IntServ model, the applications must define the desired route for their packets and must also reserve the required resources before transmitting the data. On the DiffServ model, there is no resource reservation. Packets receive transmission priorities according to pre-defined service classes; packets of the same class receive similar treatment. Traffic engineering main objective is to optimize the network resources. MPLS is a packet routing mechanism within in which packets are labeled; packet routes are defined based on both labels and the constraints imposed by the applications.

To solve the problem of real-time communication, policing traffic mechanisms can also be implemented. Policing mechanisms control and monitor the network flows to guarantee the requested QoS. If in a given moment the network do not provides the required QoS, control measures are taken, such as: packet delay, discard or marking.

Several policing mechanisms haven been proposed. Among the most cited on the literature, there are the Leaky Bucket (Butto et. al., 1991) and its variants (Berger, 1990), the peak counter, and the windowbased mechanisms. Each of these mechanisms use some of the following parameters:

a) the average rate, which defines the transmission average rate (packets per time interval) by which a flow can inject packets or bytes into the network;

b) the peak rate, which upper bounds the quantity of packets or bytes that can be sent in a short time interval;

c) size of blast, which upper bounds the maximum number of packets or bytes that can be sent instantly.

Interoperability among several systems from many suppliers is possible through the use of the TCP/IP protocol. TCP is a protocol from the transport layer of Internet TCP/IP architecture. This protocol is connection-oriented and offers a reliable end-to-end file transfer service. It is responsible for inserting the application messages into the transport datagram, for organizing the arrival of datagrams and also for resending lost datagrams. TCP was designed to work using the connectionless network service offered by the IP protocol. Therefore, it does not require a reliable network service, being responsible for the recovery of damaged, lost or doubled data and also for re-ordering data delivered out of order by the network protocol.

Nagle apud (Lima and Fonseca, 2002), on the initial phase of Internet, were one of the first to find that routers were vulnerable to congestion collapse. Such phenomenon, despite being rare, can occur when traffic is intense and the network is congested, provoking the discard of packets in the routers and resultant retransmissions, thus increasing round trip time (RTT). When packets are discarded, the resources that were used by them are wasted. Besides, when these packets are retransmitted, new resources are allocated.

Jacobson, who developed a mechanism that forces machines involved on TCP communication to reduce their transmissions of packets if there is congestion, introduced the first solution proposed for congestion problems within TCP/IP networks. This mechanism is composed of two algorithms: Slow Start and Congestion Avoidance, which are implemented together in practice. Since then, the efficiency of congestion control and prevention mechanisms was improved, with the proposal of two other algorithms: Fast Retransmit and Fast Recovery. Such mechanisms, composed by the four algorithms (TCP Reno, TCP Vegas, TCP Sack, TCP New Reno), is 
part of the TCP congestion control (Lima and Fonseca, 2002).

Another approach is proposed in (Liberatore, 2002), where a middleware regulates the network access through a polling scheme. This middleware is built on top of RTP/UDP/IP, which can also provide flexible support for fault tolerance, error recovery, and bandwidth monitoring. This middleware controls every network application, avoiding collision problems and controlling application priorities.

On (Kweon, et. al., 2000) a traffic smoothing solution is introduced, which is implemented between the UDP or TCP layer and the Ethernet MAC layer. This mechanism gives priority to realtime (RT) packets over packets of applications that do not require real-time communication. Through this mechanism, a reduction of the network collision rate was evidenced, thus guaranteeing RT applications.

(Dias et al., 2003) employ the Lagrangean relaxation to optimize IP network traffic over MPLS technology; in this work, traffic-engineering tasks were modeled as a math-programming problem. The use of Lagrangean relaxation with heuristics was more efficient than the standard routing algorithms. (Pedattela et al. 2003) introduce a Framework that aims to guarantee end-to-end QoS for the Internet through the use of a set of MPLS and DiffServ models and also through the use of a management software based on configuration policies.

\section{Methodology of ANALYSis}

The implementation of pervasive distributed systems based on the widespread use of components ubiquitously incorporated into the physical world, imposes advanced research work in the domains of convergence between communications, computing, and control.

The proposed work fits completely in the scope of this particular area. Its main objective is to propose and analyze solutions enabling the implementation of network-based control systems supported by accessuncontrolled networks (such as packet-based networks). Within the framework of this project are envisaged both the cases of control systems supported by local networks (LANs), or remote control systems that involve the use of public data communication networks.

The fundamental assertion of this project is that QoS architectures will be the appropriate architectures to support the implementation of NCS, especially when supported by access-uncontrolled networks (i.e., communication environments subject to unknown disturbances).

In order to achieve the targeted objective, a detailed state-of- the-art study is being done on both:

a) Methodologies for support of real-time communication, either through local networks, or public data communication networks.

b) Methodologies for the implementation of network-based control systems.
At the same time, an experimental platform to test network-based control systems is being developed (Figure 1) based on the following components:

a) a 2D table for the swinging of a ball;

b) a $2 \mathrm{D}$ position acquisition system for tracking the position of the ball on the table, based on a realtime image processing system;

c) A real-time control and actuation system suited to control the $2 \mathrm{D}$ position of a ball.

The interconnection between the acquisition system and the control system will be made through a selectable communication system, which will allow experimental tests to the performance of different communication methodologies, either for local networks (LANs) or for public data communication networks. In order to reproduce the traffic patterns from the several services found on a real system, a disturbance generator interconnected to the system under test will be used. Such disturbance generator will produce real system workloads and, for that, trace files will be used containing the actual features of the system in which the system to be controlled will be added to.

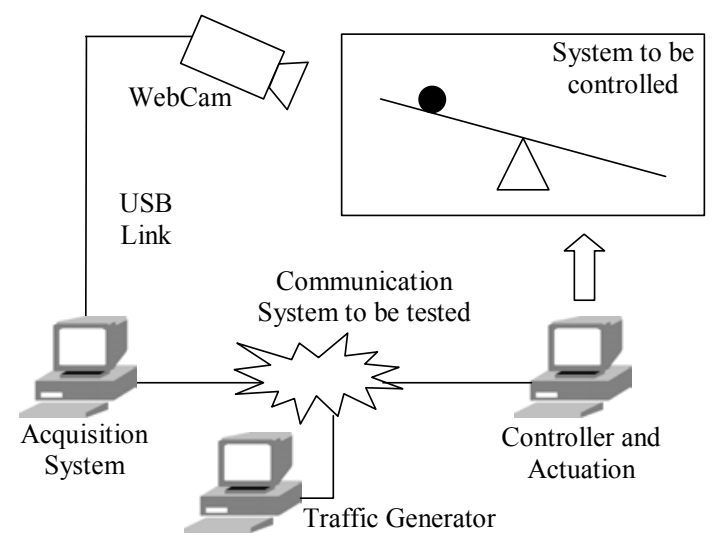

Fig. 1. Experimental platform to test network-based control systems.

According to the mentioned real-time support technologies, the communication system to be tested will be modified. This way, innovative real-time communication mechanisms, appropriate to be implemented on communication protocols traditionally used on either local networks (LANs) or on public data communication networks, will be proposed, tested and analyzed. Thus, with the use of such experimental platform, the impact of different communication methodologies on the quality of service offered to a specific control application will be evaluated. One of the major research targets is the evaluation of which communication parameters have a decisive impact on the control stability.

\section{CONCLUSION}

The advent of ubiquitous, distributed computation, communication and sensing systems will have a huge impact in applications where computer systems strongly interact with the environment, enabling the true implementation of the pervasive distributed computing paradigm. 
Control systems are fundamental elements for the construction of complex computer systems characterized by such close interaction with the exterior environment. The implementation of network-based feedback control systems, usually referred as Network-based Control Systems (NCS), will have a strong impact on the implementation of the pervasive distributed computing paradigm.

Moreover, considering the innovative domains of application where computer systems strongly interact with the environment, it is our firm believe that there will be strong pressure for the implementation of NCS supported by public data communication networks. However, current technologies are still not able to robustly support control tasks by means of public data communication networks. Thus, there is demand for advanced design methodologies able to implement such kind of NCS.

We argue that there are two kinds of approaches to solve this problem: either new control approaches are developed enabling the consideration of the inherent temporal unpredictability of public data networks; or new communication methodologies are proposed for supporting real-time communications on public data networks.

In this paper, an on going-project was presented (still without experimental results), where it was pointed out that QoS architectures are the appropriate architectures for supporting communication on public data networks, in a temporally predictable way. An experimental platform was also presented, where the selected methodologies for the support of real-time communication will be tested.

\section{REFERENCES}

Berger, A. (1990). Performance Analysis of a Rate Control Throttle Where Tokens and Jobs Queue. In: Proceedings of IEEE INFOCOM, pp. 30-38.

Butto, M., E. Cavallero and A. Tonietti (1991). Effectiveness of the Leaky Bucket Policing Mechanism in ATM Networks. In: IEEE JSAC, pp. 335-342, vol. 9, no. 3 .

Dias, A. D., E. Camponogara, J.M. Farines, R. Wilrich, A. Campestrini (2003). Otimização Lagrangeana em Engenharia de Tráfego para redes IP sobre MPLS. In: 21 . Simpósio Brasileiro de Redes de Computadores - SBRC 2003, pp. 475-490.

Ferrari, D. and D. Verma (1990). A Scheme for Real-Time Channel Establishment in Wide-Area Networks. In: IEEE Journal on Selected Areas of Communications, pp. 368-379.
Granville, L.Z. Gerenciamento Integrado de QoS em Redes de Computadores (2001). Tese de Doutorado em Ciência da Computação, Universidade Federal do Rio Grande do SUL UFRGS. Porto Alegre. Brasil. 159 f.

IETF (2002), INTERNET ENGINEERING TASK FORCE. Disponível em: http://www.ietf.org. Abril 2002.

Kandlur, D., K. Shin and D. Ferrari (1994). RealTime Communicaiton in Multi-Hop Networks. In: IEEE Transactions on Parallel and Distributed Systems, vol. 5, no. 10, pp. 10441056.

Kweon, S., K. Shin and G. Workman (2000). Achieving Real-Time Communication over Ethernet with Adaptive Traffic Smoothing. In: 6th IEEE Real Time Technology and Applications Symposium (RTAS 2000), pp. 90100.

Liberatore, V. (2002). Scheduling of Network Access for Feedback-based Embedded Systems. In: Quality of Service over Next Generation Internet, SPIE ITCom 2002, pp. 73-82.

Lima, M.A.E., N.L.S. Fonseca (2002). Controle de Tráfego Internet. In: $20^{\circ}$. Simpósio Brasileiro de Redes de Computadores - SBRC 2002, pp. 187249.

Martí, P., J. Fuertes, G. Fohler and K. Ramamritham (2001). Jitter Compensation for Real-Time Control Systems. In: Proceedings of the 22nd IEEE Real-Time Systems Symposium (RTSS'01), pp. 39-48.

Murray, R., K. Åström, S. Boyd, R. Brockett and G. Stein (2003). Future Directions in Control in an Information-Rich World. To appear in IEEE Control Systems Magazine. Disponível em: http://www.cds.caltech.edu/ murray/papers/200 3a mur+03-csm.html

Pedatella, R., E. Madeira, M. Magalhães (2003). Um Framework para Obtenção de QoS fim-a-fim na Internet. In: 21 ${ }^{\circ}$. Simpósio Brasileiro de Redes de Computadores - SBRC 2003, pp. 491-506.

Schmidt, D.C. et al (2000). Developing Next Generation Distributed Application with QoS Enabled DPE Middleware, In: IEEE Communications, pp. 112-123.

Wang, Z. (2001). Internet QoS: Architectures and Mechanisms for Quality of Service. Morgan Kaufmann Publishers.

Zheng, Q. and K. Shin (1998). Fault-Tolerant RealTime Communication in Distributed Computing Systems. In: IEEE Transactions on Parallel and Distributed Systems, vol. 9, no. 5, pp. 470-480. 